\title{
Working with the complexities of adolescent mental health problems: applying Time-limited Adolescent Psychodynamic Psychotherapy (TAPP)
}

\author{
Stephen Briggs ${ }^{1}$, Monique Maxwell², Amanda Keenan ${ }^{3}$
}

\begin{abstract}
:
This article discusses the application in a CAMHS setting of a distinctive intervention for adolescent mental health difficulties, Time-limited Adolescent Psychodynamic Psychotherapy (TAPP). TAPP has been developed specifically for working with adolescents and the characteristic developmental and psychosocial complexities they present to mental health services. It is widely recognised that supporting the developmental process in adolescence is central to therapeutic interventions and the therapeutic aim of TAPP is to enable recovery of the capacity to meet developmental challenges. The key factors of TAPP are described, including the formulation and working with a developmental focus, the therapeutic stance, working with transference and counter-transference, working with time limits, and the emphasis on engagement of adolescents in therapy in TAPP. The experiences of introducing and developing TAPP in the CAMHS service are discussed with two brief and one extended case examples and this leads to a discussion of the kinds of outcomes achieved. It is concluded that TAPP is a key and relevant intervention for adolescents in complex and vulnerable situations; further work will be undertaken to continue its application in these settings and to formally assess outcomes.
\end{abstract}

\section{Key Words:}

adolescent mental health, , complexity, adolescent development, CAMHS, timelimited psychotherapy

\section{Introduction: engaging adolescents in psychotherapy}

Short-term or time-limited psychotherapies have been demanded increasingly by mental health services, and in recent years applied in a number of formats for

\footnotetext{
${ }^{1}$ Professsor of Social Work, University of East London

${ }^{2}$ Child and Adolescent Psychotherapist, Leicester Partnership NHS Trust

${ }^{3}$ Lead Child and Adolescent Psychotherapist, Leicester Partnership NHS Trust
} 
psychoanalytically based therapeutic work with adolescents, including MBT (Rossouw and Fonagy 2012), DIT (Lemma et al 2011), Short-term Psychodynamic Psychotherapy (STPP) (Trowell et al 2007, Midgley et al 2013). In the current contexts for practice of evidence based practice, and austerity, brief therapies are attractive - although perhaps for the wrong kinds of reasons (see Lemma et al 2011, chapter 1) and increasingly they are being applied in a range of contexts within health services and elsewhere. On the other hand, many proponents of brief or time-limited models extol their virtues, stemming back to the original claims made by Malan (2003, page xi) for the particular techniques developed by practitioners, notably Habib Davanloo, and recent empirical evidence also supports the effectiveness of short term psychodynamic psychotherapy (Abbass et al 2013).

In this burgeoning field of time-limited therapy for adolescents, these models have often been adapted from models initially developed for adults. The Malan approach to Intensive Short term Psychodynamic Psychotherapy influenced the early development of TAPP (Time-limited Adolescent Psychodynamic Psychotherapy). Subsequently, influenced by the experiences of applying the model to adolescents, TAPP now places a core emphasis on the aim of effecting change to the process of developmental in adolescence, across the whole adolescent age range, up to and beyond 18 years. Additionally, TAPP focusses on addressing the complex psychosocial vulnerabilities and predicaments that adolescents frequently present to mental health services. Cottrell and Kroom (2005) pointed out a decade ago, that a gap was appearing in child and adolescent mental health services between the evidence for effectiveness in interventions - based on single issues, primarily depression - and the complex nature of the problems young people present, "with troubling predicaments, not neat diagnoses" [Cottrell and Kroom 2005 page 115].

There are compelling reasons for emphasising the need for adolescent-oriented therapies for working with these 'troubling predicaments'. Firstly, there are longstanding concerns about difficulties in engaging those adolescents most in need in psychotherapy, and, secondly, clinicians working with young people are well aware of the need to adapt techniques for adults (and indeed children) to make effective therapeutic contact with young people; thus when working with adolescents, the therapist is positioned differently, emotionally_and mentally, and this stems from the 
relational tensions and ambiguities that are the consequences of the profound developmental processes in adolescence, tensions which involve complex, emergent and mixed feelings, thoughts and actions about separateness and intimacy, independence and dependence; ambiguous communications about defensiveness and growth can become easily confused and confusing (Waddell 2006).

Recognising, and working with the adolescent's anxieties at the start of therapy can be effective in increasing the quality of engagement. Some common anxieties include uncertainty about the nature of the therapeutic process, and anxieties aroused by the clinic setting. Attending to these anxieties can help make sense of adolescents' experiences of beginning therapy. The widely perceived difficulties of engagement of adolescents may have contributed to the failure, before now, to develop adolescentcentred models of time-limited therapy (Briggs and Lyon, 2011). One aspect of this challenge of engagement is a difference in expectations and perceptions between (adult) professionals and young people caused by the juxtaposition of two time frames (Shefler 2000): for some adolescents, even a short-term therapy can be experienced as entrapping, whilst for others any hint of a thought of an ending can be experienced as an abandonment or an affront to the developing adult ego.

Taking on an adolescent perspective implies accepting the ambiguities in communications, stemming from ambivalence and the delicacy of the adolescent's fledgling sense of separateness and independence. Young people can be thought of as occupying a boundary state between being a child in the family and an adult in the world (Waddell 1998); being able to occupy this boundary domain - and also to escape from it by going back into the family or getting out into the world - requires flexibility in the internal world, as well as a reliable social world, with access to figures supportive of development. Adolescents tend to arrive for therapy stuck on one side or the other of this boundary; some can hardly bear to stay in a room with a therapist, whilst others arrive at the first session almost as if they have brought their suitcase ready to move in. For the former the question is how can s/he be helped to stay a bit longer and, for the latter, how to manage transitions and struggles over separating. With both kinds of adolescent positioning it is important to assess actions as much as words: often this amounts to watching where young people's feet take them, and what they move towards and away from. The two combined - feet and words -can give a good picture of 
the quality of ambivalence the young person is contending with, and there can be moment to moment oscillation between these positions.

\section{Mental health difficulties in adolescence}

There is, appropriately, both anxiety and concern about the prevalence of mental health difficulties in adolescents, with intervention in adolescence having the potential to prevent long-term mental health disorders. But recent studies show that whilst half of all lifetime mental disorders begin before 14 and 70\% by age 24 (HMG/DH 2011 page 50), most do not persist into adulthood:

"Episodes of adolescent mental disorder often precede mental disorders in young adults. However, many such disorders, especially when brief in duration, are limited to the teenage years, with further symptom remission common in the late 20s" (Patton et al 2014).

These authors identify the key factor for persistence of mental health problems into adulthood as their-duration in adolescence: the longer the duration, the more likely they will persist into adulthood (Patton et al 2014). Clinicians in CAMHS, and other services working with young people with adolescent mental health difficulties, are aware on a daily basis that some of their adolescent patients are engaged in a chronic and longterm relationship with the service, with frequent re-referrals. On the other hand, there are others who appear to resolve seemingly intractable problems almost unexpectedly. The research evidence thus supports both these clinical perceptions of repeated presentation but also recovery.

Adolescents presenting with mental health difficulties can require intensive interventions, which may not need to be long-term, to enable resolution of mental health problems. They also require a process of 'gathering up' when in crisis or caught up in apparently intractable and interminable mental health struggles. Many of the latter are linked with social vulnerabilities, including having experienced abuse, being in care, being teenage parents, offenders, seeking asylum, being homeless, and/or discriminated against in BME communities (DCSF/DH 2008). Adolescents in these complex, vulnerable contexts, are referred to CAMHS with mood disorders, episodes of self-harm, instabilities in relationships with others, and difficulties with emotional regulation. Often, multiple services are involved and there can be a lack of integration 
between them [O'Reilly et al 2013]. Thus these young people are assessed as presenting some of the highest risks, and they are likely to have poorer outcomes [Kessler et al 2010].

\section{Adolescent development in current social contexts}

Psychoanalytic thinking has understood the importance of the process of development in adolescence as creating an often intense emotional experience offering possibilities for change and growth; a 'second chance' (Blos 1967). However, the radically changed and changing social and economic contexts for young people are generating new and different psychological tasks and requiring specific qualities to negotiate transitions that have become extended, less structured, 'disembedded' and more uncertain, as has been widely discussed (Briggs 2009, Cote 2014, Furlong and Cartmel 2007). Particularly, pathways into adulthood have become more complex. Whilst psychoanalytic thinking has richly theorised the development from puberty into adolescence, there has been less written to explain how adolescents move into adulthood.

These transitions create deeply contradictory situations: young people may be psychologically ready to leave home, take up a career, enter a partnership but continue to depend on parental support. It is more realistic to see adolescents moving into adulthood piecemeal or in uneven ways, becoming positioned as 'more adult' in particular domains- such as relationships, employment, becoming a parent (Briggs 2008). Recognition of diverse pathways, based on social inequalities, is found in the concept of 'fast' and 'slow' track routes into adulthood (Jones 2005), and these have different consequences and associated difficulties. The slow track route involves a delayed transition to adulthood, longer time spent (often through the third decade) in semi-independent relatedness to parents (including financial dependency) and deferred taking up of work or career roles. The uncertainties of the slow track route can arouse anxieties and be difficult to bear; the psychological tasks of 'becoming-a-subject' have thus needed to be revised to fit the time scales and the partial independence. On the other hand, fast track routes can lead to premature taking up of adult roles - often by the most disadvantaged young people - with risks of social exclusion, marginalisation, lack of training, work and education. 
Interventions for adolescents therefore need to fulfil some key criteria: they need to be 'adolescent-centred' to facilitate engagement; they need to recognise the diversity -and multiplicity - of pathways into adulthood, the vulnerability generated by the intense process of adolescent change, and also the potential this creates for growth through development, including in more disadvantaged and apparently entrenched situations. Thus addressing adolescent mental health difficulties requires relating these factors to both psychological and social aspects through attention to the emotional and relational aspects of experience.

\section{What is TAPP?}

It is widely recognised historically (Anderson and Dartington 1998) and currently (Midgley et al 2013) that supporting the developmental process is central to therapeutic interventions with adolescents. Time-limited Adolescent Psychodynamic Psychotherapy (TAPP) takes this view, and enhances it, so that the aim of TAPP is to identify and work with a developmental focus in each individual case. The focus is negotiated specifically for the context and experiences of each individual adolescent, paying attention to the individual's narrative of themselves, and their social contexts. In general terms, the focus usually relates to an aspect of the adolescent developmental process and emerging subjectivity (subjectivation), involving increasing emotional and mental (and sometimes geographic) separation from parental figures, and the ownership of the adult sexual body, one’s own thoughts, drives and impulses (Laufer 1985, Briggs 2008).

TAPP proposes that through the therapeutic focus on a significant area of developmental difficulty and/or disturbance, for a time-limited period, the young person can be enabled to recover the capacity to meet developmental challenges and/or have this capacity strengthened. This focus involves attention placed on and intervention with maladaptive, stuck, regressive or anti-developmental aspects and also potentially developmentally supporting and sustaining aspects. The approach formulates problem behaviours, states of mind and feelings, and symptoms in terms of disturbances to the adolescent developmental process, understood to involve interactions between internal, interpersonal, and social aspects. The aim is therefore to restore or strengthen the potential for developmental growth, rather than providing a 'cure' for all psychopathology; this is the therapeutic goal. Therapeutic structures of 
time and a focus on a developmental aspect can help young people manage development and transitions in their social and cultural contexts. The accurate identification of the developmental focus for intervention for each adolescent involves working with a powerful constellation of anxieties, defences, and internal and social relationships, including early core relatedness. Thus the model is a deep psychotherapy and requires skilled handling by experienced clinicians who can work with the demands of being in receipt of intense emotions with infantile roots, and be able to make sense of these to initiate change and development. TAPP takes a psychoanalytic approach to therapeutic relatedness, including focus on the transference and counter-transference and working in depth. The power of the model stems from the handling of transference and counter-transference and the time-limits, which introduces the concept of separation from the outset, so that a mirror of the adolescent developmental processes of separation, transition and growth is held in the therapeutic relationship and structure.

\section{The development of TAPP/structure}

TAPP was developed in the Tavistock Clinic's Adolescent Department, initially by Louise Lyon, who initiated and led the Brief Therapy Workshop to creatively introduce a timelimited model, which was initially reported using the nomenclature of TPP-A (Briggs 2010, Briggs and Lyon 2011, Briggs and Lyon 2012). TAPP is an individual psychotherapy for adolescents with an overall structure of -usually - a 20 session therapy, involving 4 sessions assessment/engagement, 16 sessions therapy working to a developmental focus agreed by therapist and adolescent patient, followed by a review 4-8 weeks after the end of therapy. Work with parents and other professionals can be facilitated alongside the TAPP therapy,

The extended assessment phase, based on the Tavistock Clinic's Adolescent Department approach to assessment and, deriving from the 'trial therapy' approach of Intensive Short -term Dynamic Psychotherapy (ISTDP) (Malan and Coughlin Della Silva 2006), aims to provide young people with an experience of the therapeutic process, and to actively engage the adolescent in identifying, with the therapist, the focus for therapy. It also provides a very brief intervention, and an opportunity to not continue in therapy (for both sides). The engagement process through repeated sessions in the assessment 
phase is one of the key features of the 'adolescent-centred' nature of TAPP. Additionally, though the therapy is then agreed for a number of weeks (usually 16) the end date is discussed in terms of a time which is relevant for the young person's life - for example, up to the end of the school year, before exams start, after exams finish, before you go to university, start work, etc; the young person is actively engaged in the contracting process. The therapy is conducted as usual in psychodynamic psychotherapy the adolescent patient brings to each session whatever is on her/his mind, and the therapist works with this within the framework of time and focus.

Recent developments in TAPP include manualisation (Briggs 2013), the development of a training for qualified and experienced psychodynamic practitioners, which is accredited by the British Psychotherapy Council, and the introduction of TAPP in different settings in the UK including CAMHS -an example of which is discussed here and practitioners working in educational settings.

\section{Therapeutic stance and therapeutic priorities}

TAPP adopts and maintains a therapeutic stance that provides a containing therapeutic space and which is thus both supportive and promoting possibilities for exploration, as with longer term psychotherapy. Support for the therapist is provided through supervision/seminar group, focussing on the meaning of the therapist's emotional experiences as these can often be intense, under the pressure of adolescent emotionality and having to retain in mind a penumbra of ambiguities. Additionally, and importantly, the therapist works with time-limits, both practically to manage the number of sessions available, and also to enable exploration of the meaning of the timelimit for the young person, and within the therapeutic interactions. It is central to TAPP that the replaying of the core developmental issues in the transference are felt and observed through the time scale, and thus the meaning of time and time limits is central to the process of change and growth.

The therapist employs an active, attentive and empathic manner to facilitate engagement with the adolescent patient, orienting to the 'in between' states of mind of the adolescent, and aiming to occupy a position from which s/he can both reach out to and contain anxieties arising from the young person's needs for emotional support from more-adult others, and also refrain from intrusive or infantilising interventions that 
may have a crushing effect on aspirant independence and separateness. The therapist attends to these issues through the way the structure of the therapy is handled and also through how the therapist relates to the patient in the sessions. In practice this means providing a space to think, and moving responsively between more adult/more childlike states as they occur within the sessions. The therapist aims therefore to not become identified by the patient as solely expecting or relating to adult like states, nor solely as a parental figure of a child-patient.

The therapist works a great deal with counter-transference experiences, feelings and thoughts, and reflection in supervision or a group setting is important to enable the therapist to retain optimum positioning, work with and make sense of the feelings and thoughts, work with the developmental focus and maintain the sense of time in the time-limited therapy. There is often a great deal of anxiety to manage. The therapist has to overcome and eschew the temptation to become caught up with an anxiety to work quickly and cover breadth. Instead, the aim is to work in depth, repeatedly returning to the focus to explore them in greater depth and thus to understand the meaning of these for development. It is important to not lose patience in the face of recurring difficulties, which can be accompanied at times by intense emotions and the risks of unhelpful and possibly self-destructive actions. Equally, it is also vital to have the capacity to spot change and growth, albeit this often appears to ambiguously suggest either development or defensiveness.

How the transference is worked with in each case is also a key consideration for reflection and supervision. Transference interpretation is used primarily to enhance working with the developmental process, and the balances - or imbalances - for the adolescent between dependence/independence and intimacy/separateness. Transference interpretation can help identify patterns and working in the room with the relationship. They can be formulated in terms of adolescent development to locate the young person's concerns and expressions in the way that he experiences the developmental process, and which address anxieties and defences. These will usually involve identification of the young person's positioning with regard to separateness and dependency on adults, ranging on the spectrum from engulfment/entanglement to withdrawal from relatedness to others. In some TAPP cases, transference interpretations are made frequently, whilst in others rarely. 


\section{Change and growth}

The emphasis in the therapeutic work is to find ways in which the adolescent developmental process can be restored, re-energised and/or freed from the constraints of pathological parts of the self, and this is accomplished through working with the developmental focus. From working with this focus, adolescents develop a sense of being more able to manage their emotional, relational and social worlds. High levels of engagement have been regularly obtained in different settings. Symptom reduction may be an outcome, and in some cases, symptoms may resolve relatively quickly. On the other hand, some adolescents, especially those who start TAPP with projective or distancing defences may find themselves becoming more aware of their difficulties, and the feelings and thoughts that accompany these. In this sense, TAPP, like many psychodynamic therapies, aims to increase awareness of distress, not reduce it (Lemma et al 2011, page 15)

TAPP does not aspire omnipotently to resolve all problems in 20 sessions, but rather to seek to provide the conditions whereby the developmental process will continue to support growth after the therapy ends. Change is evidenced through a greater engagement with relational and emotional experiences, so the young person becomes more 'subject of' the internal, interpersonal and social factors and forces affecting him/her, accompanied by richer, more coherent narratives of the self and an awareness, perhaps accompanied by ironic reflection, of patterns of defensiveness. Adolescent patients thus become more aware of and interested in their development: "one of the outcomes is the young person's engagement with the continuous project of themselves, the development of their subjectivity, and awareness of their emotionality" (Briggs and Lyon 2011 p423). It follows that for some young people, an outcome for TAPP can be an interest in undertaking further therapy, either soon after the TAPP intervention or at a later date, and this will be explored in the therapy, or at the follow up review.

\section{Application in CAMHS}

TAPP was introduced by a child psychotherapy team in a CAMHS service in 2013, where the aim was to offer a relatively brief, time-limited therapy for young people who have complex presentations and who have often experienced previous and multiple interventions, often across services. The decision to introduce TAPP followed an audit 
(Keenan et al 2013) which found that all referred adolescents had multiple complexity factors, evidenced through clinical need (diagnoses), environmental factors and contact with other agencies. Of the sample audited ( $n=24)$, only $33 \%$ children resided with both parents, $50 \%$ had a documented history of early abuse/neglect, $50 \%$ had experience of domestic violence in the household and $58 \%$ had one or both parents with a diagnosed mental health condition. Half had a history of self-harm, and $90 \%$ had two or more prior CAMHS treatments.

To help address numbers of cases awaiting treatment and to effect earlier intervention a psychotherapist was engaged to specifically focus on short-term work. Following this, the team engaged with the TAPP model, including a reflective seminar. Recognising the importance of outcome research for psychotherapy in the current contexts, we have embarked on a process of systematically evaluating TAPP outcomes, and this will be reported in a separate article. Here we can comment on the numbers of young people referred to TAPP, undertaking and completing assessments, beginning and completing treatment. For the initial 18 months, between March 2013 and October 2014, 28 cases of young people between 15 and 17 years began assessment for TAPP. We are finding that there are good rates of completion of both assessment and treatment; 22/25 (88\%) completed assessment ${ }^{4}$, and 15/17 (88\%) completed treatment. We have also found that attendance at sessions is high, with all young people in treatment attending 12 or more of the 16 sessions (75\%). Indicatively, patients report high levels of satisfaction at the end of assessment and treatment. Satisfaction with TAPP is found in the scores and comments of the Experience of Service Questionnaire (ESQ); patients record being listened to (100\%), having worries taken seriously (100\%) and $80 \%$ would recommend TAPP for a friend (80\%).

We have also seen that some young people do not proceed from assessment to treatment, as can be expected in a model that encourages negotiation and opting in to treatment, on the one hand, and a detailed assessment of needs on the other hand. 7/22 $(32 \%)$ did not continue from assessment to treatment for a range of reasons: 4 declined an offer of treatment, for 2 it was agreed treatment was not needed and for 2 it was assessed that TAPP was not suitable at that time.

\footnotetext{
${ }^{4} 3 / 28$ young people were currently in the process of assessment at the time of writing
} 
All these cases fulfilled the criteria for 'complexity', with diagnosable mental health difficulties, extensive experiences of previous treatments in CAMHS, and/or in other health services and social care. For some of these young people, therapists applied TAPP to engage with extremely stuck development and, alongside other professionals, worked intensively with these young people to find ways of generating more hopeful developmental pathways.

Three case examples will be used to illustrate this range of issues, and the process of therapy, including the developmental focus, and the assessed qualities of change. The cases illustrate different aspects of complexity; longstanding family relationship difficulties and a resort to suicidal solutions; an apparent sexual perversion in a young man, who had lived in a violent home in childhood, and a young woman, also with a very difficult childhood, who became a teenage mother. Two of these are presented as vignettes, and the third in an extended format illustrates some of the key therapeutic processes in TAPP.

Vignette 1: The first vignette illustrates the role of TAPP following a referral of a young person after an episode of self-harm. Josie 5 , 15 years, was referred by a CAMHS psychiatrist who had seen her following an overdose of paracetamol. In assessment, Josie expressed a sense of shock and contrition about the overdose, but she also presented as emotionally frozen, as though traumatised (Briggs et al 2012). Her suicidal state appeared to have arisen as a way out of an impasse in development in which she appeared enmeshed with her mother and caught up in an endless series of arguments with her. The developmental formulation was that Josie's enmeshment with mother restricted her adolescent development, especially in terms of becoming more separate from her mother, developing her own more independent thoughts and feelings, and having more involvement in her peer relationships.

The constellation of depression, including problems in mourning the absence of her birth father, provided a difficult and intense emotional environment and thus she turned to self-destructive solutions as development - and life itself - seemed impossible.

\footnotetext{
${ }^{5}$ All names have been changed to preserve confidentiality
} 
Josie accepted the offer of TAPP therapy to work on becoming more separate, and working toward freeing herself in her development through exploring her feelings.

Josie engaged well in her therapy, and she began to claim her adolescent development, a sign of which was that she recognised her $16^{\text {th }}$ birthday, which occurred during the therapy, as a positive landmark and at this time began a relationship with a boyfriend. Development on the side of life thus began to appear in place of the restricted, deathly quality of development with which she presented initially. The work on the developmental focus of the enmeshed relationship with mother led to her finding more freedom and space in her relationships. There was no repetition of self-harm and at follow-up the risks appeared sufficiently reduced to not offer further interventions immediately, though Josie expressed a wish for further therapy in the future.

Vignette 2: Peter, a young man of 17, generated high levels of anxiety in his GP and CAMHS when his father discovered Peter's involvement in what these professionals described as perverse behaviour. Father was very angry, afraid and concerned about his 'deviant' son, though he was also preoccupied with his relationship with a new partner, who was resentful about Peter's presence. Initial discussion about how to proceed included referral to an organisation specialising in work with perverse and dangerous young people, however it was agreed by the multi-disciplinary team that initially a child psychotherapy assessment should be undertaken, with a view to either TAPP or a specialist referral. Separately, another professional offered consultations with his father and the other members of the family.

Peter engaged in the assessment, attending the sessions and sharing a detailed narrative of his life, which enabled understanding of his behaviour as a response to adverse early experiences and current turbulence in his family. He felt 'freaky' and his behaviour thus augmented his low self-esteem and lack of satisfactory peer relationships. Peter accepted the offer of TAPP with the focus on making sense of his childhood experiences, how these became eroticised and thus intruded on his adolescent development, and this in turn led to his sense of rejection and exclusion. In contrast to the reactive and provocative relationships in his family past and present, the reflective qualities of the therapeutic relationship, were important for Peter to gather a 
sense of himself as less damaged and more self-accepting. He attended most of his sessions, and his review follow up session, and at the end of his therapy he felt positively about his experience of TAPP; he felt more accepted at home and more at ease in his college environment. The anxieties generated by his referral had been significantly reduced, and referral to a specialist agency was not needed.

\section{Case example}

This case provides a more detailed account of the therapeutic processes in TAPP when working with young people who present complex difficulties. Beth illustrated the impact of social vulnerabilities and the pressures of a 'fast-track route' towards adulthood (Jones 2005). She became pregnant and had a baby at the age of 17 , and her GP, worried about her anxiety and postnatal depression referred her to CAMHS. Beth's parents were baby Kelly's main carers, and though there is evidence that teenage mothers face stigma, lack of informal support and the unavailability of relevant and appropriate information (Boath et al 2013), in Beth's case, the key relationships with her parents were highly conflictual and this appeared to be a major contributor to her current turmoil.

A very first and striking impression of Beth was that she seemed to have astigmatism (a squint), which gave the impression she couldn't focus. I [MM] found myself wondering if Beth could see the baby. Beth came to her first assessment alone, that is, without her baby, and began by saying it was difficult for her to attend her sessions, as it conflicted with her college commitments. Concretely therefore Beth brought an 'adolescent' aspect to this first meeting, rather than a 'mother'. Her life seemed torn in two directions; her face lit up when she spoke about the baby, but she spoke openly too about how she felt much too young to be a mother, and that if she had become aware that she was pregnant earlier in her pregnancy (she found out at 20 weeks) she would have had a termination. When it was clear it was too late for this she said her life spiralled into turmoil. She presented with strongly conflicting feelings, that her baby was 'everything to her', on the one hand, but, on the other hand, she wanted to be a 'proper teenager' and be in college, get the qualifications she needs and the future she wanted for herself. 
It was clear from what Beth said that her mother did most of the caring for her baby, and Beth appeared wildly unrealistic about being available to meet the needs of her baby herself. She said she felt good when she was directly caring for Kelly, but she had also taken a part-time job; she wanted her baby to know she had worked hard to provide for her, and she felt guilty that her parents had already provided so much for Beth. There seemed to be a real tangle about dependence and independence, which Beth projected on to her baby, for example when she talked about ways in which she wanted Kelly to be independent that were not age appropriate. Anxiety was generated in me about Beth's lack of realism as a parent, and as her own childhood grievances surfaced there appeared to be two babies to think about.

Beth described a difficult and conflictual childhood home, and Beth said she did not want Kelly to go through "family issues" impacting on her schooling and her life, as had been her own experience. She wanted Kelly to know that she could always come and talk to her, and that she would understand; Beth agreed, when I asked her, that she had not felt understood as a child. Beth also spoke about Kelly as being a "such a good listener". I asked her what she meant and she said that she told Kelly everything, all her worries and how her day has been. I felt a strong pull to ask Beth to bring Kelly to her therapy and it was confusing to think about whose infantile needs were present.

In the third assessment session I introduced a discussion about how to proceed after the 4 assessment sessions and whether it would feel helpful to come for therapy, and for how long, leading to making an offer of TAPP to think about her really difficult situation and how to find a pathway through. Beth spoke with passion -and distress- about her predicament, showing determination to keep going with her college course and parttime job and fury about becoming a mother. I said she felt things were hugely unfair and that she was struggling to come to terms with what had happened and the fact that she was now a mum - and also how very angry she feels at times about what had happened to her.

In the melée of her current crisis and difficult childhood past, it seemed that the only horizon was one in which some structure could be given to thinking about these issues and to see what could be thought about, or not, and what path Beth could find to take 
from here. Hence the 16 sessions of TAPP appeared a reasonable, practical framework for Beth at this time, in a landscape of some confusions and uncertainties. It was difficult to contain the intense feelings that had arisen through the event of Beth's pregnancy and her conflicts about being an adolescent and being a mother, together with the regrets and grievances regarding the present, and the past.

Poignantly, in the $4^{\text {th }}$ assessment session, while confirming her acceptance of the offer of TAPP, Beth said she had her first pay cheque, and that she would use some of the money to buy presents for Kelly, or, on second thoughts, she would buy a winter coat for herself. This appeared to restate Beth's dilemma; on the one hand she aimed to be a providing parent, but this led to the need to provide for herself and to overcome her own dependent needs - which she wanted to hurry her daughter out of too. The winter coat also appeared to have a meaning of an insulating, or protective, layer to help with her vulnerabilities, which appeared to go through her with all the chill of winter.

\section{Treatment sessions:}

Beth attended her TAPP therapy sessions, making considerable efforts to fit in her attendance along with her other commitments. She used her sessions to express her anxieties, to 'get things off her chest', continued with her college course and by the $10^{\text {th }}$ session she reported she had caught up with her assignments. She felt considerable pride in this achievement, which was driven by complicated motivation: she felt robbed of her future by getting pregnant, and she persisted with college to get qualifications "so that both she had Kelly had a future". There is an element here of Beth attempting to do something about her fate (Bollas 1991) and her determination to not succumb to the problems that follow from the 'fast track' route to adulthood.

On the other hand, her therapy continued to be dominated by a maelstrom of conflicts, primarily in Beth's interpersonal and social worlds. Conflicts between Beth and her parents escalated and Beth brought her very low feelings to her therapy, which she expressed at one point as a wish to sleep forever, though when we discussed this she did not have active suicidal plans. Concern about Kelly's welfare and her care led to social care involvement; CAMHS was involved in the ensuing multi-agency discussions. Beth was helped to face her painful feelings and expectations towards her parents and 
to recognise that they would not be met; this appeared to catalyse her own wish to mother her child. Gradually within the therapy and in her contacts with other services, Beth obtained advice and representation and she applied for a place in a mother and baby unit.

Her TAPP therapy helped Beth contain her anxieties so that she could begin to work through her grievances about her childhood and, somewhat relieved from these, she became more able to work out what she wanted for herself and her baby; she become more empowered and active in supporting herself including working with other professionals to apply for the place in a mother and baby unit; having taken this position, her relationship with parents improved also. She was less depressed, better able to manage her relationship with her parents and at the end of her therapy she was expectantly awaiting a place in a mother and baby unit. She remained vulnerable and CAMHS provided follow up until this place became available. Throughout she kept up her college attendance and work. Noticeably, by the end of the treatment her squint had gone; both eyes were focused, giving an embodied indication that for Beth things had come into focus.

\section{Discussion}

This article has discussed the application of TAPP in a CAMHS service. TAPP is a distinctive time-limited psychodynamic psychotherapy, which has been developed specifically for working with adolescents and the characteristic complexities they present to mental health services. The therapeutic aim of TAPP is to enable recovery in young people in difficulty of the capacity to meet developmental challenges and/or have this capacity strengthened. The key factors of the TAPP model, as described above, include the formulation and working with a developmental focus, the therapeutic stance, working in depth and working with time limits. A central feature is the emphasis on active engagement of young people in the therapeutic process through attending to the emotional experience of entering therapy, repeated meetings in the assessment to provide an experience of therapy, and discussion of the focus and duration of therapy.

An account is given of the first 18 months of the introduction and development of TAPP in a CAMHS service. Two vignettes and a more detailed case example are provided to illustrate the process of therapeutic intervention using TAPP and this leads to a 
discussion of the kinds of outcomes achieved. These demonstrate clarification of complex difficulties, reduction of symptoms and containment of extreme anxieties. These examples illustrate the role of TAPP in harnessing developmental potential, in individualised ways leads to less destructive and more benign development.

The case examples indicate some aspects of the range of TAPP interventions working with complex adolescent cases in CAMHS. They show that TAPP is a relevant intervention in these cases, where the initial referrals indicated risks, uncertainties and diverse vulnerabilities, and where the clinical presentations suggested that a holistic focus and a structured time-limited therapy make sense of these 'troubled predicaments' and provide an opportunity for engagement and exploration of the many factors, past and present, internal and external that contribute to the adolescents' current difficulties. The developmental focus of TAPP, which is based on a contemporary psychosocial understanding of the adolescent developmental process, holds together and provides a clear rationale for the intervention, which is perceived by the adolescent patients as relevant and a positive approach to their difficulties. The introduction of TAPP into this CAMHS service has offered a practical and dynamic approach to working these cases, which can seem otherwise hard to allocate to an appropriate intervention. Further work will continue to apply TAPP in this setting and to assess outcomes formally, using quantitative measurements, at the end of treatment and for longer-term outcomes.

\section{References}

Abbass, A., Rabung, S., Leichsenring, F., Refseth, J., Midgley, N. (2013) Psychodynamic Psychotherapy for Children and Adolescents: A Meta-Analysis of Short-Term Psychodynamic Models, Journal of The American Academy Of Child \& Adolescent Psychiatry, 52, 8, 863-875

Anderson, R., \& Dartington, A. eds. (1998) Facing It Out: Clinical Perspectives on Adolescent Disturbance, London, Duckworths/Tavistock Clinic Series

Boath, E., Henshaw, C., Bradley, E. (2013) Meeting the challenges of teenage mothers with postpartum depression: overcoming stigma through support, Journal of Reproductive and Infant Psychology, 31, 4, 352-369 
Blos, P. (1967) 'The Second Individuation Process of Adolescence'. Psychoanalytic Study Journal of Social Work Practice of the Child, 22, 162-186,

Bollas, C. (1991) Forces of Destiny: Psychoanalysis and Human Idiom, London, Free Association Books

Briggs, S. (2008) Working with Adolescents and Young Adults; a contemporary psychodynamic approach, Basingstoke, Palgrave Macmillan

Briggs, S. (2009) Risks and opportunities in adolescence: understanding adolescent mental health difficulties, Journal of Social Work Practice, 23, 1, 149-164

Briggs, S. (2010) Time limited psychodynamic psychotherapy for adolescents and young adults., 24, 2, 181-196

Briggs, S. (2013) Manual for Time Limited Adolescent Psychodynamic Psychotherapy (TAPP)

Briggs, S. and Lyon, L. (2011) A developmentally focussed time-limited psychodynamic psychotherapy for adolescents and young adults: origins and applications, Revue Adolescence, no.76, 415-434

Briggs, S. and Lyon, L (2012) Time limited psychodynamic psychotherapy for adolescents and young adults, in Lemma, A. ed. Contemporary Developments in Adult and Young Adult Therapy, The work of the Tavistock and Portman Clinics, London, Karnac

Côté, J. (2014) Youth Studies: Fundamental Issues and Debates, Basingstoke, Palgrave Macmillan

Cottrell, D. \& Kroom, A. (2005) Growing Up? A History of CAMHS (1987-2005) Child and Adolescent Mental Health, 10, 3, 111-117

DCSF/DoH (2008) Children and young people in mind: the final report of the National CAMHS Review http://www.bpmhg.org.uk/wp-content/uploads/2012/10/camhsreview.pdf

Furlong, A. \& Carmel, F. (2007) Young People and Social Change; new perspectives, Buckingham, Open University Press, $2^{\text {nd }}$ edition 
HMG/DH (2011) No Health Without Mental Health: A Cross-Government Mental Health Outcomes Strategy for People of All Ages www.dh.gov.uk/mentalhealthstrategy

Jones, G. (2005) The thinking and behaviour of young adults, http://www.wirrallearningpartnership.org/downloads/Policies,plans\%20,publications /thninking\%20behaviour\%20of\%20young\%20people\%200DPM\%20Nov\%2005.pdf

Keenan, A., Smyth, P. and Thomaidis-Zades, K. (2013) Complexity Factors in the Presentation of Children and Adolescents in Child Psychotherapy: A baseline service evaluation of child psychotherapy cases, Leicester Partnership NHS Trust

Kessler, R., McLaughlin , K., Green , J., Gruber, M. et al (2010) Childhood adversities and adult psychopathology in the WHO World Mental Health Surveys, British Journal of Psychiatry, 197: 378 - 385

Laufer, M. (1985) The Suicidal Adolescent, London, Karnac

Lemma, A., Target, M., Fonagy, P. (2011) Brief Dynamic Interpersonal Therapy ; a Clinician's Guide, Oxford, Oxford University Press

Malan (2003) Foreword, in Osimo, F. Experiential Short-term Dynamic Psychotherapy: A Manual, Bloomington, IN, First Books

Malan, D. \& Coughlin Della Silva, P. (2006) Lives Transformed; a revolutionary method of dynamic psychotherapy, London, Karnac

Midgley, N., Cregeen, S., Hughes, C., Rustin, M. (2013) Psychodynamic Psychotherapy as Treatment for Depression in Adolescence, Child and Adolescent Psychiatric Clinics of North America 22, 67-82

O’Reilly, M., Vostanis, P., Taylor, H., Day, C., Sreet, C. \& Wolpert, M. (2013) Service user perspectives of multiagency working: a qualitative study with children with educational and mental health difficulties and their parents, Child and Adolescent Mental Health, 18, 4, 202-209,

Patton, G. Coffey, C., Romaniuk, H., Mackinnon A. Carlin, J. et al (2014) The prognosis of common mental disorders in adolescents: a 14-year prospective cohort study, The Lancet, 383, 9926, 1404-1411 
Rossouw, T., \& Fonagy, P. (2012) Mentalization-Based Treatment for Self-Harm in Adolescents: A Randomized Controlled Trial, Journal of The American Academy Of Child \& Adolescent Psychiatry, 51, 12, $1304-1313$

Shefler G. 2000 Time limited psychotherapy with Adolescents, Journal of Psychotherapy Practice and Research 9, 88-99

Trowell, J., Joffe I, Campbell J, Clemente C, et al (2007) Childhood depression: a place for psychotherapy; An outcome study comparing individual psychodynamic psychotherapy and family therapy, European Child and Adolescent Psychiatry, 16, 3, 157-67.

Waddell, M. (1998) Inside Lives: Psychoanalysis and the Growth of the Personality. (London, Duckworths/Tavistock Clinic Series)

Waddell, M. (2006) Narcissism- an adolescent disorder? Journal of Child Psychotherapy $32,1,21-34$ 